\title{
A Genome-Wide Association Study Identifying SVEP1 Variant as a Predictor of Response to Tolvaptan for Cirrhotic Ascites
}

Yasuhito Tanaka ( $\nabla$ ytanaka@med.nagoya-cu.ac.jp)

Kumamoto University: Kumamoto Daigaku https://orcid.org/0000-0002-2473-6966

Hideto Kawaratani

Nara Medical University School of Medicine Graduate School of Medicine: Nara Kenritsu Ika Daigaku Igakubu Igakuka Daigakuin Igaku Kenkyuka

\section{Hiromi Sawai}

University of Tokyo Graduate School of Medicine Faculty of Medicine: Tokyo Daigaku Daigakuin Igakukei Kenkyuka Igakubu

\section{Masaya Onishi}

Nagoya City University Graduate School of Medical Sciences and Medical School: Nagoya Shiritsu

Daigaku Daigakuin Igaku Kenkyuka Igakubu

\section{Tomomi Kogiso}

Tokyo Women's Medical University: Tokyo Joshi Ika Daigaku

Noritomo Shimada

Otakanomori Hosipital

Haruki Uojima

Kitasato University School of Medicine: Kitasato Daigaku Igakubu

\section{Tomoaki Nakajima}

Sapporo Kosei General Hospital: Sapporo Kosei Byoin

\section{Naoki Matsumoto}

Nihon University School of Medicine Graduate School of Medicine: Nihon Daigaku Igakubu Daigakuin Igaku Kenkyuka

\section{Kenichi Ikejima}

Juntendo University School of Medicine Graduate School of Medicine: Juntendo Daigaku Igakubu Daigakuin Igaku Kenkyuka

\section{Toru Ishikawa}

Saiseikai Niigata Hospital: Saiseikai Niigata Byoin

\section{Shuji Terai}

Niigata Daigaku

\section{Hiroyuki Motoyama}

Osaka City University: Osaka Shiritsu Daigaku 


\section{Atsumasa Komori}

Nagasaki Medical Center Clinical Research Center: Kokuritsu Byoin Kiko Nagasaki Iryo Center Rinsho Kenkyu Center

\section{Noboru Hirashima}

National Hospital Organization Nagoya Medical Center: Kokuritsu Byoin Kiko Nagoya Iryo Center

\section{Satoru Saito}

Yokohama City University School of Medicine Graduate School of Medicine: Yokohama Shiritsu

Daigaku Igakubu Daigakuin Igaku Kenkyuka

\section{Yuichiro Eguchi}

Saga University: Saga Daigaku

\section{Masanori Nojima}

University of Tokyo Institute of Medical Science: Tokyo Daigaku Ikagaku Kenkyujo

\section{Yosuke Kawai}

National Center for Global Health and Medicine: Kokuritsu Kenkyu Kaihatsu Hojin Kokuritsu Kokusai Iryo Kenkyu Center

\section{Masakuni Tateyama}

Kumamoto University: Kumamoto Daigaku

\section{Hitoshi Yoshiji}

Nara Medical University School of Medicine Graduate School of Medicine: Nara Kenritsu Ika Daigaku Igakubu Igakuka Daigakuin Igaku Kenkyuka

\section{Research Article}

Keywords: blood urine nitrogen (BUN), genome-wide association study (GWAS), Tolvaptan, non-responder, Polydom, FOXC2, liver cirrhosis, hepatic ascites, transcriptional analysis, RNA-Seq

Posted Date: April 28th, 2021

DOI: https://doi.org/10.21203/rs.3.rs-388508/v1

License: (c) (i) This work is licensed under a Creative Commons Attribution 4.0 International License. Read Full License 


\section{Abstract}

Background and Aims: Tolvaptan, an orally active vasopressin V2-receptor antagonist, has been used for patients with difficult-to-treat ascites in Japan. In this study, we conducted a genome-wide association study (GWAS) in the Japanese population to identify genetic variants associated with tolvaptan's efficacy for patients with hepatic ascites.

Methods: From 2014 through 2018, genomic DNA samples were obtained from 550 patients who were treated with tolvaptan. Of those, 80 cases (non-responder; increase of body weight [BW]) and 333 controls (responder; > $1.5 \mathrm{~kg}$ decrease of BW) were included in the GWAS and replication study.

Results: GWAS showed 5 candidate SNPs around the miR818, KIAA1109, and SVEP1 genes. After validation and performing a replication study, an SNP (rs2991364) located in the SVEP1 gene was found to have a significant genome-wide association $\left(\mathrm{OR}=3.55, \mathrm{P}=2.01 \times 10^{-8}\right)$. Univariate and multivariate analyses showed that blood urea nitrogen (BUN) and SVEP1 SNP were significantly associated with the response $(O R=1.03, p=0.02$ and $O R=4.24, p<0.0001$, respectively). Based on a prediction model of logistic regression analysis in a population with the rs2991364 risk allele, the failure probability (= exp (score: $\left.22.234+\mathrm{BUN}^{\star} 0.077+\mathrm{Na}{ }^{\star}-0.179\right)(1+\exp ($ score $))$ was determined for the detection of nonresponders. Assuming a cutoff of failure probability at $38.6 \%$, sensitivity was $84.4 \%$, specificity was $70 \%$ and AUC was 0.774 .

Conclusion: SVEP1 rs2991364 was identified as the specific SNP for the tolvaptan response. The prediction score can identify tolvaptan non-responders and help to avoid a lengthy period of futile treatment.

\section{Introduction}

Hepatic ascites, the accumulation of fluid in the abdominal cavity due to liver disease, is a difficult condition to treat. In European guidelines, high-dose diuretics (160mg furosemide, spironolactone $400 \mathrm{mg}$ ) are used for uncontrolled hepatic ascites (1). However, Japanese patients are usually intolerant to high-dose conventional diuretics (furosemide and spironolactone) because of dehydration or hyponatremia. Since December 2013, tolvaptan, an orally active vasopressin V2-receptor antagonist, has been used in Japan for patients with ascites that are difficult to treat with conventional diuretics (2). Tolvaptan suppress the expression of aquaporin (AQP)-2 and inhibits water reabsorption in the renal collecting ducts. Tolvaptan does not stimulate sodium channels, unlike other diuretics, and increases free water excretion without affecting urinary sodium levels (3). Japanese guidelines recommend administering 20 to $80 \mathrm{mg}$ furosemide and/or 25 to $100 \mathrm{mg}$ spironolactone (4) for mild ascites. When ascites is uncontrolled by conventional diuretics, tolvaptan is administered at a dose of $3.75 \mathrm{or} 7.5 \mathrm{mg}$. Tolvaptan was first reported to increase serum sodium levels safely and effectively in patients with euvolemic and hypervolemic hyponatremia in a study that explored ascending doses of tolvaptan (SALT-1 and SALT-2 studies) in 2006 (5). Other studies have also demonstrated the efficacy and safety of 
tolvaptan (6). In a Japanese multicenter retrospective study, responders to tolvaptan were defined as patients who had body weight (BW) loss of $1.5 \mathrm{~kg} /$ week which reflect the improvement of ascites volume and symptoms (7). Since this paper was published, BW loss of $1.5 \mathrm{~kg} /$ week criterion has been applied to determine the efficacy of tolvaptan. About two-thirds of patients showed an extreme increase in urine volume and/or decrease in BW. Conversely, roughly one-third of patients showed no increase in urine volume and/or decrease in BW. Several studies showed that high blood urine nitrogen (BUN) or high urine osmolality can worsen the treatment response $(8,9)$, and that creatine (Cre) does not necessarily influence the response to tolvaptan.

The genome-wide association study (GWAS) method has been used to predict the treatment response, such in the analysis of IL28B variants strongly associated with the response to pegylated-interferon (IFN) plus ribavirin (RBV) therapy for chronic hepatitis $C$ patients (10). We hypothesized that the response to tolvaptan differs based on clinical characteristics and host genetics. The reason for the difference in the effect of tolvaptan with respect to clinical characteristics has been discussed previously $(6,7)$. In this study, to determine whether to extend administration to tolvaptan non-responders in the hope that they will eventually respond, we conducted a GWAS in the Japanese population to identify genetic variants associated with tolvaptan's efficacy in patients with difficult-to-treat ascites, and to identify nonresponders to tolvaptan.

\section{Material And Methods}

\section{Patients}

This study was conducted nationwide in Japan from 2014 through 2018. The protocol was registered to the clinical trials registry managed by the University Hospital Medical Information Network in Japan (registration no. UMIN000025905). Genomic DNA samples were obtained before and after tolvaptan administration from 550 patients who had been treated with tolvaptan for hepatic ascites at each of the participating hospitals (40 hospital liver units with hepatologists). Responders to tolvaptan was defined as those who had a greater than 1.5-kg decrease in BW after 1-week tolvaptan treatment; and nonresponders, as those with an increase in BW after 1-week tolvaptan treatment. A total of 80 cases (nonresponders) and 333 controls (responders) were included in the GWAS and replication study, and 137 borderline patients whose BW change after 1-week tolvaptan treatment was $0 \mathrm{~kg}$ to $-1.49 \mathrm{~kg}$, were excluded from the study. To identify genetic variants associated with the response to tolvaptan in patients with cirrhotic ascites, we performed a cohort GWAS recruiting 181 patients (25 cases and 156 controls) from 2014 to 2016 for SNP candidates with $p<10^{-4}$. A replication study recruiting 232 patients (55 cases and 177 controls) from 2017 to 2018 was also performed.

The study protocol was conducted in accordance with the ethical guidelines of the Declaration of Helsinki and was approved by each of the participating Institutional Ethics Review Committees for our human genome projects. Written informed consent was obtained from all individual participants.

\section{Evaluation of GWAS}


Genomic DNA was extracted from peripheral blood leukocytes using a standard method. In the GWAS stage, we genotyped 253 patients using the Affymetrix Axiom Genome-Wide ASI 1 Array (Thermo Fisher Scientific) according to the manufacturer's instructions, and determined the genotype calls of 600,307 SNPs using Genotype Console v4.2.0.26 software. All samples used for genotyping passed a Dish QC > 0.82 and we excluded 1 sample with an overall call rate $<97 \%$. We recalled the remaining 252 samples with Genotyping Console software. The average Dish QC for 252 samples was $0.953(0.877-0.988)$ and the average call rate reached 98.87 (97.02-99.86). All genotyped samples passed a heterozygosity check and identity by descent testing. A principal component analysis found 2 outliers that could be excluded by the Smirnov-Grubbs test, and we showed that all the remaining samples formed a single cluster with the HapMap Japanese (JPT) samples but not with the Han Chinese (CHB), Northern and Western European (CEU), and Yoruba (YRI) samples. We then applied the following thresholds for SNP quality control in data cleaning: SNP call rate $\geq 95 \%$, minor allele frequency (MAF) $\geq 5 \%$, and Hardy-Weinberg equilibrium (HWE) $P$ value $\geq 0.001$. A total of 411,709 SNPs on autosomal chromosomes passed the quality control filters and were used for subsequent GWAS.

\section{Statistical analysis}

Details of the prediction model based on a logistic regression model in which the backward elimination $(\mathrm{P}<0.05)$ method was applied after including age, sex, weight, Child-Pugh classification, platelet, \%PT, albumin, AST, ALT, total bilirubin, BUN, Cre, Na, etiology (HBV, HCV, alcohol, and NASH). Receiver operating characteristics (ROC) analysis was used to assess the prediction ability of the model. All analyses were carried out using SPSS for Windows version 25.0 (IBM, Armonk, NY, USA).

In the GWAS (including genome-wide imputation data) and replication study, the chi-square test was applied to a 2-by-2 contingency table in the allele frequency model. The odds ratio (OR) and the confidence interval $(\mathrm{Cl})$ were calculated using the major alleles as references. We considered $\mathrm{P}<5 \times 10^{-8}$ as the threshold for genome-wide significance in the combined analysis.

In the replication stage, we selected 134 SNPs with $P$ values $<10^{-5}$ and linkage disequilibrium (LD) $<0.9$ from the results of the chi-square test in the GWAS using genome-wide imputed data. We additionally selected 49 SNPs located on the functionally interested genes. DigiTag2 assay and TaqMan SNP genotyping assay (Applied Biosystems, Foster City, CA, USA) were used to confirm the genotypes at each SNP. We genotyped 80 cases and 333 controls to validate the GWAS results and for the replication study.

\section{Results}

\section{Baseline characteristics of enrolled patients}

A total of 413 patients were analyzed in this study. Table S1 shows the baseline clinical characteristics of the patients (269 men and 144 women). The mean age was 67.2 years (range: $37-97$ years). Etiologies of the patients were viral hepatitis B in 38 patients, viral hepatitis $C$ in 147 patients, heavy alcohol consumption in 126 patients, non-alcoholic steatohepatitis in 50 patients, autoimmune hepatitis in 9 
patients, primary biliary cholangitis in 24 patients, and other causes in 28 patients. A total of 163 patients (39.5\%) had hepatoma. The baseline Child Pugh classification was A in 5 patients, B in 193 patients, and $C$ in 215 patients. Adverse events occurred in 86 patients. The events included thirst in 43 patients (50.0\%), hepatic coma in 21 patients $(24.4 \%)$, dehydration in 9 patients $(10.5 \%)$, and other (hypernatremia, muscle cramps, and eruption etc.) in 13 patients (15.1\%). There were $333(80.6 \%)$ patients who were responders to tolvaptan and 80 patients $(19.4 \%)$ who were non-responders. Table 1 showed the comparison of clinical characteristics by the tolvaptan's response. Comparing between 80 non-responders and 333 responders, there were statistical differences in BMI $(P=0.02)$, presence of HCC $(P=0.04)$ and $\mathrm{BUN}(P=0.005)$.

\section{Genetic variants associated with response to tolvaptan}

We conducted a GWAS using 25 cases (non-responders) and 156 controls (responders) by analyzing 411,709 autosomal SNPs. Figure S1 shows a genome-wide view of the SNP association data based on allele frequencies. There were 80 SNPs with P values $<10^{-4}$ in the GWAS. Of the 80 SNPs, 20 and 5 SNPs showed $P$ values $<10^{-5}$ and $<10^{-6}$, respectively. The 5 candidate SNPs were identified around miR818 (rs16827413: $P=5.97 \times 10^{-7}$ and rs10800602: $P=8.69 \times 10^{-8}$ located on chromosome $1 \mathrm{q} 32.1$ ) and KIAA1109 (rs3108399: $P=5.84 \times 10^{-8}$ on chromosome 4 q27) and SVEP1 (rs9299186: $P=7.46 \times 10^{-7}$ and rs4978937: $P=7.46 \times 10^{-7}$ on chromosome 9 q31.3).

\section{Imputation-based GWAS and replication study}

We performed genome-wide imputation-based GWAS in order to find additional candidate SNPS associated with the response to tolvaptan (Figure S2). There were 2,127 SNPs with P values $<10^{-4}$ in the imputation-based GWAS, and 497 SNPs with P values $<10^{-5}$. Of the 497 SNPs, 134, 102, and 87 SNPs showed $L D<0.9,<0.7$, and $<0.5$, respectively. For validation and replication, we selected 192 SNPs including 134 SNPs with P values $<10^{-5}$ and LD $<0.9,9$ SNPs on AXIOM ASI 1 array, and 49 SNPs located on the functionally interested genes. The original GWAS set of 181 samples ( 25 cases and 156 controls) and an independent set of patients (55 cases and 177 controls) were genotyped and used in a subsequent replication analysis. Of the candidate SNPs, 3 (rs2991364, rs9299186, and rs4978937 on SVEP1 intron region) were validated and consistent associations were observed between the GWAS set and replication set (Table 3). One SNP showed a genome-wide significant association (rs2991364: OR = $3.55, P=2.01 \times 10^{-8}$ ) using the combined set (80 cases and 333 controls) (Table 2).

\section{Risk factors for response to tolvaptan}

The results obtained by multivariate logistic regression analysis are shown in Table 3. Univariate analysis showed that BUN and SVEP1 SNPs (rs2991364) were significantly associated with the response to tolvaptan, and multivariate analysis showed that BUN and SVEP1 SNPs were also associated with the response to tolvaptan $(O R=1.03, p=0.02$ and $O R=4.24, p<0.0001$, respectively). Table $S 2$ shows that the highest sum of sensitivity and specificity of BUN was 0.51 and 0.71 , respectively. Using the best cutoff value of BUN (22.4), the area under the curve (AUC) of BUN was 0.64 , and the $95 \% \mathrm{Cl}$ was $0.57-$ 
0.70. The highest sum of sensitivity and specificity of SVEP1 SNP was 0.43 and 0.85 , respectively. The AUC of SVEP1 was 0.64 , and $95 \% \mathrm{Cl}$ was $0.58-0.70$. The highest sum of sensitivity and specificity of combination of SVEP1 SNP and BUN was 0.60 and 0.70 , respectively. The AUC for the combination of SVEP1 SNP and BUN was 0.69, and $95 \% \mathrm{Cl}$ was $0.62-0.76$. The difference in AUC between SVEP1 SNP alone and the combination of SVEP1 SNP plus BUN was significant $(p<0.01)$ (Figure S3). These results suggest that the combination of BUN and SVEP1 SNP could predict the response to tolvaptan more accurately and identify non-responders.

\section{Constructing a prediction model for detecting non- responders}

In the non-risk allele group, $86.0 \%$ of the patients achieved a treatment response, which is sufficient to consider the risk to be low. On the other hand, the percentage of unsuccessful treatment cases was around $40 \%$ in the risk allele group (red area), and the number of successful cases was as high as $60 \%$ (Fig. 1a). Therefore, it is important to conduct a detailed risk assessment in the risk allele group. A logistic model, using the variable reduction method, structured the prediction model based on BUN and $\mathrm{Na}$, and the predictive score $($ score $)=22.234+\mathrm{BUN} * 0.077+\mathrm{Na} *-0.179$ was calculated. The probability (failure probability $=\exp ($ score $) /(1+\exp ($ score $))$ was identified for detecting non-responders (Fig. 1b). Assuming a cutoff of failure probability of $38.6 \%$, sensitivity was $84.4 \%$, specificity was $70 \%$, and the AUC was 0.774 (Fig. 1C). When the failure probability was more than $38.6 \%$, the treatment failure rate was $64.3 \%$, while the treatment response was observed in $87.5 \%$ of the patients with a failure probability $\leq 38.6 \%$ (Fig. 2). There was a statistically significant difference between the groups $(p<0.001)$.

\section{Functional analysis of SNPs rs2991364 in SVEP1 gene}

To determine the effect of the rs2991364 genotype on SVEP1 mRNA expression levels, we evaluated the expression quantitative trait loci (eats) of rs2991364 using the Genotype-Tissue Expression (GITEX Release V8) project dataset via the website gtexportal.org. (11). In the GITEX data, rs2991364 minor CT/TT-allele variants led to lower SVEP1 gene expression in brain tissue [brain - cerebellar hemisphere: $\mathrm{P}$ $=1.40 \times 10^{-6}$, brain - cerebellar: $\left.\mathrm{P}=2.00 \times 10^{-6}\right]$.

We next used a using public database to determine whether SNP rs2991364 influences the expression of SVEP1 downstream signal genes. We analyzed WGS and RNA-seq data from hepatitis C virus-associated cancer patients in ICGC - RIKEN, Japan (LIRI-JP) cohorts (12). After searching for SNP rs2991364 in germline data from HCC patients, we selected SNPs rs2991364 major TT-allele patients $(n=37)$ and minor CT-allele patients $(n=10)$, and compared the gene expression level of adjuvant liver tissue between TT-allele and minor CT-allele patients (Fig. 3a). SVEP1 expression was downregulated in the minor CTallele group (Fig. 3b) $\left[\mathrm{P}=6.70 \times 10^{-4}\right]$. SVEP1 is known to deposit around lymphatic vessels and upregulates Forehead box protein C2 (FOXC2) expression through integrin alpha 9 (ITGA9), angiopoietin2 (ANGPT2) and tyrosine kinase with immunoglobulin-like and epidermal growth factor (EGF) like domain $1 / 2$ (TIE-1/2) receptors, thereby facilitating lymphatic vascular remodeling (13). Importantly, the expression levels of these SVEP1 downstream signaling genes were significantly downregulated along 
with the decrease in SVEP1 expression in the minor CT-allele group (Fig. 3c) [ITGA9: $P=0.011$, ANGPT2: $P=0.025$, FOXC2: $P=0.015]$. These results indicate that SNPs rs2991364 can influence the expression of SVEP1 and its downstream signal genes, resulting in vascular network fragility (Fig. 3d).

\section{Discussion}

In this study, we found SVEP1 SNPs correlated most closely with the response to tolvaptan. This is the first report showing a strong correlation at a genome-wide level of significance between a genetic variant (SVEP1) and the response to tolvaptan. The SVEP1 gene is located on 9q32, spans $214 \mathrm{~kb}$ of genomic DNA, and consists of 48 exons, encoding a secreted multi-domain protein which harbors sushi (named also complement control protein; CCP), von Willebrand factor (VWF) type A, EGF, and pentraxin-domain motifs (14). SVEP1 is an extracellular matrix protein involved in lymphatic vessel remodeling and plays a critical rule in epidermal differentiation (13). Previously known as Polydom, SVEP1 is also a high-affinity ligand for integrin a9b1. Polydom knockout mice show severe edema and die immediately after birth as a result of respiratory failure due to dysfunction of fluid drainage; these mice also fail to undergo remodeling and formation of collecting lymphatic vessels (13). SVEP1 is expressed in heart, lung, skeletal tissue, placenta, stomach, intestine, stromal osteogenic tissues and so on. A recent report showed that missense variants of SVEP1 were significantly related to coronary arterial disease (15).

With respect to clinical data, only BUN was identified as a response marker based on multivariate analysis (Table 3), as previously reported $(6,8)$. This result showed that renal dysfunction, especially caused by dehydration, may worsen the response to tolvaptan. On the other hand, the SVEP1 SNP (rs2991364), which was associated with SVEP1 expression levels, would influence the vulnerability of lymphatic vessels, which could lead to fluid retention. The combination of BUN and SVEP1 SNP showed a stronger correlation with the response to tolvaptan compared with BUN or SVEP1 SNP alone. Previous reports showed that various parameters were associated with the tolvaptan response, including BUN (6, 8), BUN/Cre (16), CRP (17), urine Na/K (18) ratio, urine osmolarity (9), urine AQP-2 (9, 17). As it was difficult to collect urine samples in this multicenter study, we could only compare BUN and BUN/Cre with previous reports. BUN showed a stronger correlation compared with BUN/Cre (data not shown); however other urinary parameters could not be evaluated.

It is important to identify tolvaptan non-responders to avoid a lengthy period of futile treatment. The PPV of the response to tolvaptan based on BUN was 0.86 , suggesting that BUN is a good predictive marker for the response to tolvaptan. However, because the NPV of the response to tolvaptan based on BUN was only 0.30 , it would be difficult to predict non-responders to tolvaptan using BUN alone. The NPV of the response to tolvaptan based on the combination of BUN and SVEP1 was 0.63 , indicating that this parameter was more accurate for predicting non-responders, but not sufficiently so for clinical practice. We next proposed an additional prediction model based on logistic regression analysis of a population with the rs2991364 risk allele. The failure probability based on Na and BUN was identified for detecting non-responders. Assuming a cutoff of failure probability of $38.6 \%$, sensitivity was $84.4 \%$, specificity was $70 \%$, and AUC was 0.774 , suggesting that patients with the risk allele and $a>38.6 \%$ probability of failure 
would be expected to have a poor treatment response. This may a good marker for identifying nonresponders to tolvaptan and avoid a lengthy period of futile treatment.

We propose the following strategy for the use of tolvaptan in clinical practice (Figure S4). When a patient has a BW decrease after 1 week of tolvaptan treatment, tolvaptan should be continued. However, when a patient has a BW increase after 1 week of treatment and is therefore a possible non-responder, SVEP1 SNP (rs2991364) should be measured. For patients without an SVEP1 SNP risk allele, measures should be employed to improve renal blood flow by reducing diuretics or performing large volume puncture with consideration of the late tolvaptan response (19). For patients with a risk allele, the prediction score should be determined. When the predictive score is over $38.6 \%(27 / 32,84.4 \%)$, suspension of tolvaptan treatment should be considered as well as a change to a different treatment option, such as large volume puncture.

Our bioinformatic analysis showed that the rs2991364 genotype affects the expression of the SVEP1 gene in the cerebellum, although the phenotype varies depending on the tissue. Furthermore, decreased SVEP1 expression caused a decrease in the expression of downstream genes. In particular, the expression level of the FOXC2 gene, which is known to play an important role in the development of the lymphatic vascular system (20-22), is reduced with the downregulation of SVEP1 expression. It has also been reported that FOXC2 expression is decreased through the ANGPT2 and TIE1/TIE2 receptor system in Polydor/SVEP1 mutant mice, causing severe edema (20), which is consistent with the results of our public data analysis. Our results indicate that in the rs2991364 risk allele group, tissue fluid is originally difficult to collect due to the weakness of vascular network remodeling, and suggest that rs2991364, which affects SVEP1 gene expression, may serve as an SNP marker for predicting the effect of tolvaptan in other types of edema, especially cardiac edema. A significant association between coronary artery disease and missense variants in the SVEP1 gene was reported based on a large-scale exome-wide association study (15). To elucidate the relationship between the tolvaptan response and rs2991364 allele in cardiac edema patients, further studies are required.

This study had several limitations. First, a limited number of SVEP1 SNP variants were studied. Second, the number of non-responders was relatively small and difficult to draw conclusions from. We excluded patients with a borderline response (BW change $-1.5<$ to $<0 \mathrm{~kg}$ ) to tolvaptan. Third, it was not possible to evaluate urine osmolarity or urine AQP-2. Lastly, all enrolled patients in this study were Japanese and further studies are needed to confirm the data in other populations.

In conclusion, we identified an association between SVEP1 SNPS and the response to tolvaptan among patients with difficult-to-treat hepatic ascites in the Japanese population. The combination of BUN and SVEP1 SNP was predictive of the response to tolvaptan, and the use of the predictive score can further help to identify non-responders and avoid prolonged use of tolvaptan in patients who will not ultimately benefit.

\section{Abbreviations}


Alb: albumin

ALT: alanine aminotransferase

AFP: alpha fetoprotein

$\mathrm{NH}_{3}$ : ammonia

ANGPT2: angiopoietin-2

AQP: aquaporin

AUC: area under the curve

AST: aspartate aminotransferase

BUN: blood urine nitrogen

BW: body weight

Cl: confidence interval

Cre: creatine

EGF: epidermal growth factor

FOXC2: Forehead box protein C2

GWAS: genome-wide association study

ITGA9: integrin alpha 9

IFN: interferon

Na: sodium

NPV: negative predictive value

NASH: nonalcoholic steatohepatitis

OR: odds ratio

PPV: positive predictive value (PPV)

PT: prothrombin time

ROC: Receiver operating characteristics 
T-bill: total bilirubin

\section{Declarations}

Data Availability: Yes

Animal Research (Ethics): not applicable

Consent to Participate (Ethics): Yes

Consent to Publish (Ethics): Yes

Plant Reproducibility: not applicable

Clinical Trials Registration: not applicable

Author Contribution: Yes (Substantial contributions to study conception and design: HK, HY, YT; substantial contributions to analysis and interpretation of the data: $\mathrm{HS}, \mathrm{MO}, \mathrm{MN}, \mathrm{YK}$; critical review of manuscript, acquisition of data: TK, NS, HU, TN, NM, KI, TI, ST, HM, AK, NH, SS, YE; final approval of the version of the article to be published: HK, HY, YT)

Conflict of Interest: Yes (Shuji Terai and Hitoshi Yoshi received lecture fees provided by Otsuka Pharm.

Funding: Yes (This study was partly supported by Research Program on Hepatitis from the Japan Agency for Medical Research and Development (AMED) [Grant Numbers Grant Numbers JP18fk0210001 and JP20fk0210048].)

\section{Acknowledgments}

We thank Dr. Etsuko livo, Kenta to Matsuura (Nagoya), Yuichi Yoshida (Iwate Medical university), Tomohiko Oohashi (Aichi medical university), Kazuma Eshima (India University), Atsushi Hosui, (OsakaRosia Hospital) Mari Kawakami (Okayama Kaiseki General Hospital), Shogo Shimizu (Gifu Prefectural General Medical Center), Masahito Shimizu (Gifu University Hospital), Hisamitsu Miyawaki (Nagasaki University), Moth Awasa (Mie University), Takuya Iwamoto (Yamaguchi University), Shigeru Nakano (Kaiseki Toyohashi Tobu Hospital), Takemoto Yoshio (Ehime University), Shoo Join (Kagoshima University), Noriyuki Katsu (Sapporo Medical University), Yoshiyuki Ueno (Yamagata University) for collecting samples, and Dr. Shintaro Ogawa for management of clinical samples.

\section{References}

1. European Association for the Study of the Liver. EASL Clinical Practice Guidelines for the management of patients with decompensated cirrhosis. J Hepatol. 2018;69:406-60.

2. Okita K, Sakaida I, Okada M, Kaneko A, Chayama K, Kato M, et al. A multicenter, open-label, doseranging study to exploratively evaluate the efficacy, safety, and dose-response of tolvaptan in 
patients with decompensated liver cirrhosis. J Gastroenterol. 2010;45:979-87.

3. Yamamura Y, Nakamura S, Itoh S, Hirano T, Onogawa T, Yamashita T, et al. OPC-41061, a highly potent human vasopressin V2-receptor antagonist: pharmacological profile and aquaretic effect by single and multiple oral dosing in rats. J Pharmocol Exp There. 1998;287:860-7.

4. Fukui H, Saito H, Ueno Y, Uto H, Obara K, Sakaida I, et al. Evidence-based clinical practice guidelines for liver cirrhosis 2015. J Gastroenterol. 2016;7:629-50.

5. Schrier RW, Gross P, Gheorghiade M, Berl T, Verbalis JG, Czerwiec FS, Orlandi C. Tolvaptan, a selective oral vasopressin V2-receptor antagonist, for hyponatremia. NEJM. 2006;355:2099-112.

6. Sakaida I, Terai S, Kurosaki M, Okada M, Hirano T, Fukuta Y. Real-world effectiveness and safety of tolvaptan in liver cirrhosis patients with hepatic edema: results from a post-marketing surveillance study (START study). J Gastroenterol. 2020;55:800-10.

7. Hiramine Y, Uojima H, Nakanishi H, Hiramatsu A, Iwamoto T, Kimura M, et al. Response criteria of tolvaptan for the treatment of hepatic edema. J Gastroenterol. 2018;53:258-68.

8. Kawaratani H, Fukui H, Moriya K, Noguchi R, Namisaki T, Uejima M, Kitade M, et al. Predictive parameter of tolvaptan effectiveness in cirrhotic ascites. Hepatol Res. 2016;47:854-61.

9. Nakanishi H, Kurosaki M, Hosokawa T, Takahashi Y, Itakura J, Suzuki S, et al. Urinary excretion of the water channel aquaporin 2 correlated with the pharmacological effect of tolvaptan in cirrhotic patients with ascites. J Gastroenterol. 2016;51:620-7.

10. Tanaka Y, Nishida N, Sugiyama M, Kurosaki M, Matsuura K, Sakamoto N, et al. Genome-wide association of IL28B with response to pegylated interferon-a and ribavirin therapy for chronic hepatitis C. Nat Genet. 2009;41:1105-9.

11. GTEx Consortium. Human genomics. The Genotype-Tissue Expression (GTEx) pilot analysis: Multitissue gene regulation in humans. Science. 2015;348:648-60.

12. Fujimoto A, Furuta M, Totoki $Y$, Tsunoda T, Kato M, Shiraishi Y, et al. Whole-genome mutational landscape and characterization of noncoding and structural mutations in liver cancer Nat. Genet. 2016;48:500-9.

13. Morooka N, Futaki S, Sato-Nishiuchi R, Nishino M, Totani Y, Shimono C, et al. Polydom is an extracellular matrix protein involved in lymphatic vessel remodeling. Circ Res. 2017;120:1276-88.

14. Gilgès D, Vinit MA, Callebaut I, Coulombel L, Cacheux V, Romeo PH, et al. Polydom: A secreted protein with pentraxin, complement control protein, epidermal growth factor and von Willebrand factor $A$ domains. Biochem J. 2000;352:49-59.

15. Myocardial Infarction Genetics and CARDIoGRAM Exome Consortia Investigators. et al. Coding Variation in ANGPTL4, LPL, and SVEP1 and the Risk of Coronary Disease. N Engl J Med. 2016;374:1134-44.

16. Kogiso T, Yamamoto K, Kobayashi M, Ikarashi Y, Kodama K, Taniai M, et al. Response to tolvaptan and its effect on prognosis in cirrhotic patients with ascites. Hepatol Res. 2017;47(9):835-44. 
17. Nakai M, Ogawa K, Takeda R, Ohara M, Kawagishi N, Izumi T, et al. Increased serum C-reactive protein and decreased urinary aquaporin 2 levels are predictive of the efficacy of tolvaptan in patients with liver cirrhosis. Hepatol Res. 2018;48(3):E311-9.

18. Komiyama Y, Kurosaki M, Nakanishi H, Takahashi Y, Itakura J, Yasui Y, et al. Prediction of diuretic response to tolvaptan by a simple, readily available spot urine Na/K ratio. PLoS One. 2017;12(3):e0174649.

19. Nakai M, Ogawa K, Takeda R, Ohara M, Kawagishi N, Izumi T, et al. Durable response without recurrence to Tolvaptan improves long-term survival. J Gastroenterol. 2020;55(12):1150-61.

20. Fatima A, Wang Y, Uchida Y, Norden P, Liu T, Culver A, et al. Foxc1 and Foxc2 deletion causes abnormal lymphangiogenesis and correlates with ERK hyperactivation. J Clin Invest. 2016;126(7):2437-51.

21. Kume T. The cooperative roles of Foxc1 and Foxc2 in cardiovascular development. Adv Exp Med Biol. 2009;665:63-77.

22. Lam EW, Brosens JJ, Gomes AR, Koo CY. Forkhead box proteins: tuning forks for transcriptional harmony. Nat Rev Cancer. 2013;13:482-95.

\section{Tables}

Table 1. Comparison of clinical characteristics by the tolvaptan's response 


\begin{tabular}{|c|c|c|c|}
\hline Variables & Non-responder $(n=80)$ & Responder(n=333) & P-value \\
\hline Age (years) & $66.8 \pm 12.4$ & $67.3 \pm 11.4$ & 0.83 \\
\hline Sex (male/female) & $55 / 35$ & $214 / 119$ & 0.73 \\
\hline Height (cm) & $161.7 \pm 9.7$ & $161.0 \pm 9.3$ & 0.58 \\
\hline Body weight (kg) & $63.3 \pm 14.9$ & $64.8 \pm 14.9$ & 0.44 \\
\hline BMI & $23.1 \pm 6.1$ & $24.9 \pm 4.9$ & 0.02 \\
\hline $\begin{array}{l}\text { Etiology } \\
\text { (B/C/Alc/NASH/AIH/PBC/others) }\end{array}$ & $10 / 26 / 24 / 12 / 3 / 2 / 7$ & $28 / 121 / 102 / 38 / 6 / 22 / 21$ & 0.27 \\
\hline Presence of HCC (yes / no) & $16 / 64$ & $147 / 186$ & 0.04 \\
\hline Child-Pugh (A / B / C) & $0 / 37 / 43$ & $5 / 193 / 215$ & 0.61 \\
\hline Serum albumin (g/dL) & $2.6 \pm 0.5$ & $2.7 \pm 0.5$ & 0.25 \\
\hline Prothrombin activity (\%) & $57.9 \pm 20.1$ & $59.8 \pm 18.2$ & 0.43 \\
\hline Total bilirubin (mg/dL) & $3.0 \pm 3.8$ & $\mathbf{2 . 7} \pm \mathbf{3 . 0}$ & 0.40 \\
\hline BUN (mg/dL) & $25.8 \pm 14.2$ & $21.5 \pm 13.2$ & 0.005 \\
\hline Serum creatine, $\operatorname{Cr}(\mathrm{mg} / \mathrm{dL})$ & $1.08 \pm 0.56$ & $0.97 \pm 0.46$ & 0.08 \\
\hline Serum sodium, $\mathrm{Na}(\mathrm{mEq} / \mathrm{L})$ & $135.2 \pm 5.7$ & $136.4 \pm 4.7$ & 0.65 \\
\hline Serum ammonia, $\mathrm{NH}_{3}(\mathrm{mg} / \mathrm{dL})$ & $58.1 \pm 34.1$ & $64.7 \pm 36.0$ & 0.32 \\
\hline $\operatorname{Serum} \operatorname{AFP}(\mathbf{n g} / \mathbf{m L})$ & $5.5(2.8-28.6)$ & $5.2(2.8-17.9)$ & 0.05 \\
\hline
\end{tabular}

Table 2. Three SNPs associated with tolvaptan's efficacy 


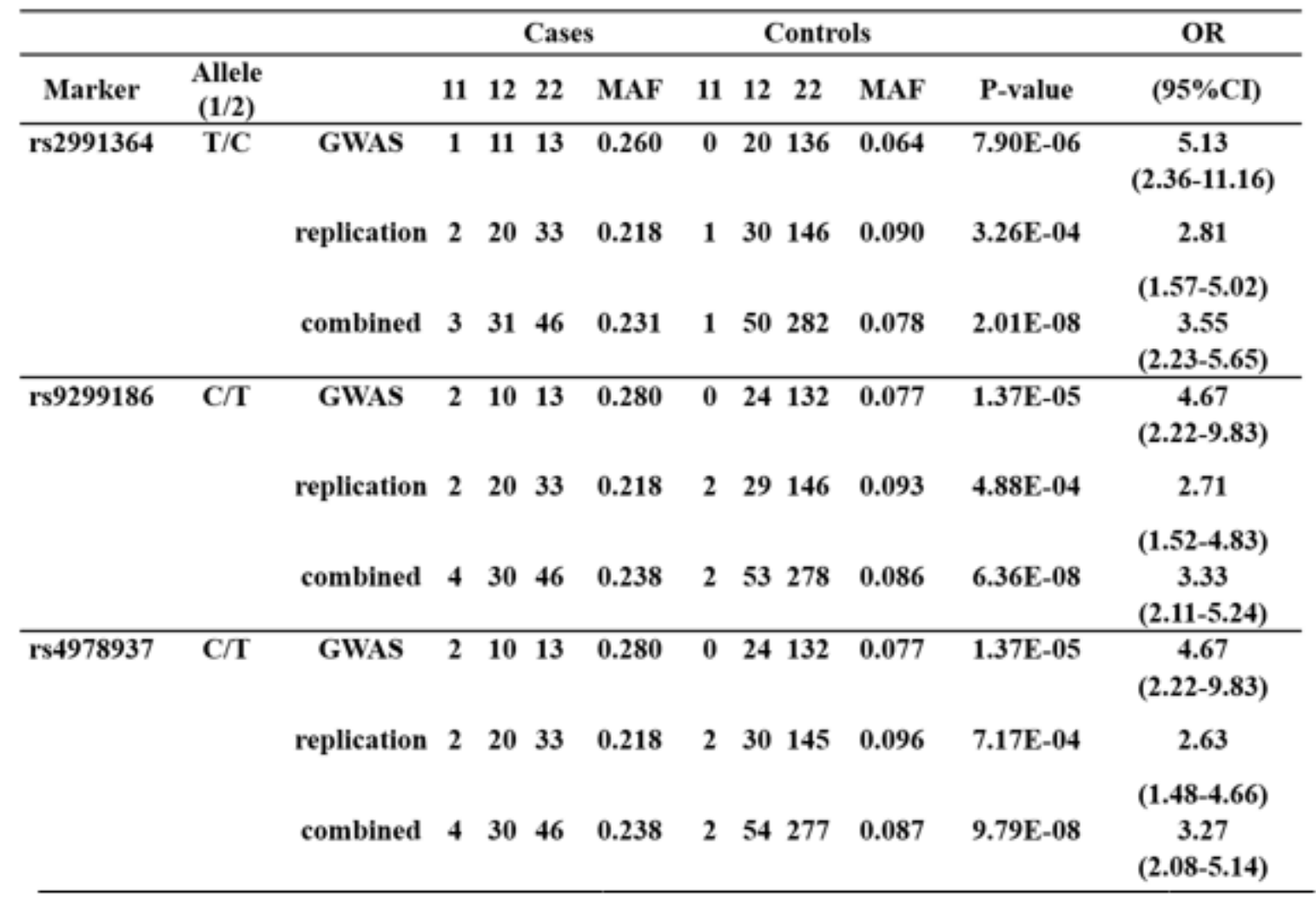

Table 3. Univariate and multivariate analysis for tolvaptan response 


\begin{tabular}{|c|c|c|c|c|}
\hline & \multirow{2}{*}{$\begin{array}{c}\text { Univariate } \\
\text { P-value }\end{array}$} & \multicolumn{3}{|c|}{ Multivariate } \\
\hline & & OR & $95 \% \mathrm{CI}$ & P-value \\
\hline Age & 0.83 & & & \\
\hline Sex & 0.73 & & & \\
\hline Child-Pugh & 0.61 & & & \\
\hline AFP & 0.05 & 1.00 & $1.00-1.00$ & 0.05 \\
\hline ALB & 0.25 & & & \\
\hline AST & 0.31 & & & \\
\hline ALT & 0.36 & & & \\
\hline BUN & 0.005 & 1.03 & $1.01-1.06$ & 0.02 \\
\hline $\mathrm{Cr}$ & 0.08 & 0.74 & $0.32-1.74$ & 0.49 \\
\hline $\mathrm{NH}_{3}$ & 0.32 & & & \\
\hline $\mathrm{Na}$ & 0.65 & & & \\
\hline Platelet & 0.90 & & & \\
\hline PT activity & 0.43 & & & \\
\hline $\begin{array}{c}\text { SVEPl } \\
\text { rs2991364 }\end{array}$ & 0.00000128 & 4.24 & 2.23-8.07 & 0.00001 \\
\hline
\end{tabular}

PT: Prothrombin, OR: odds ratio, Cl: confidence interval

\section{Figures}




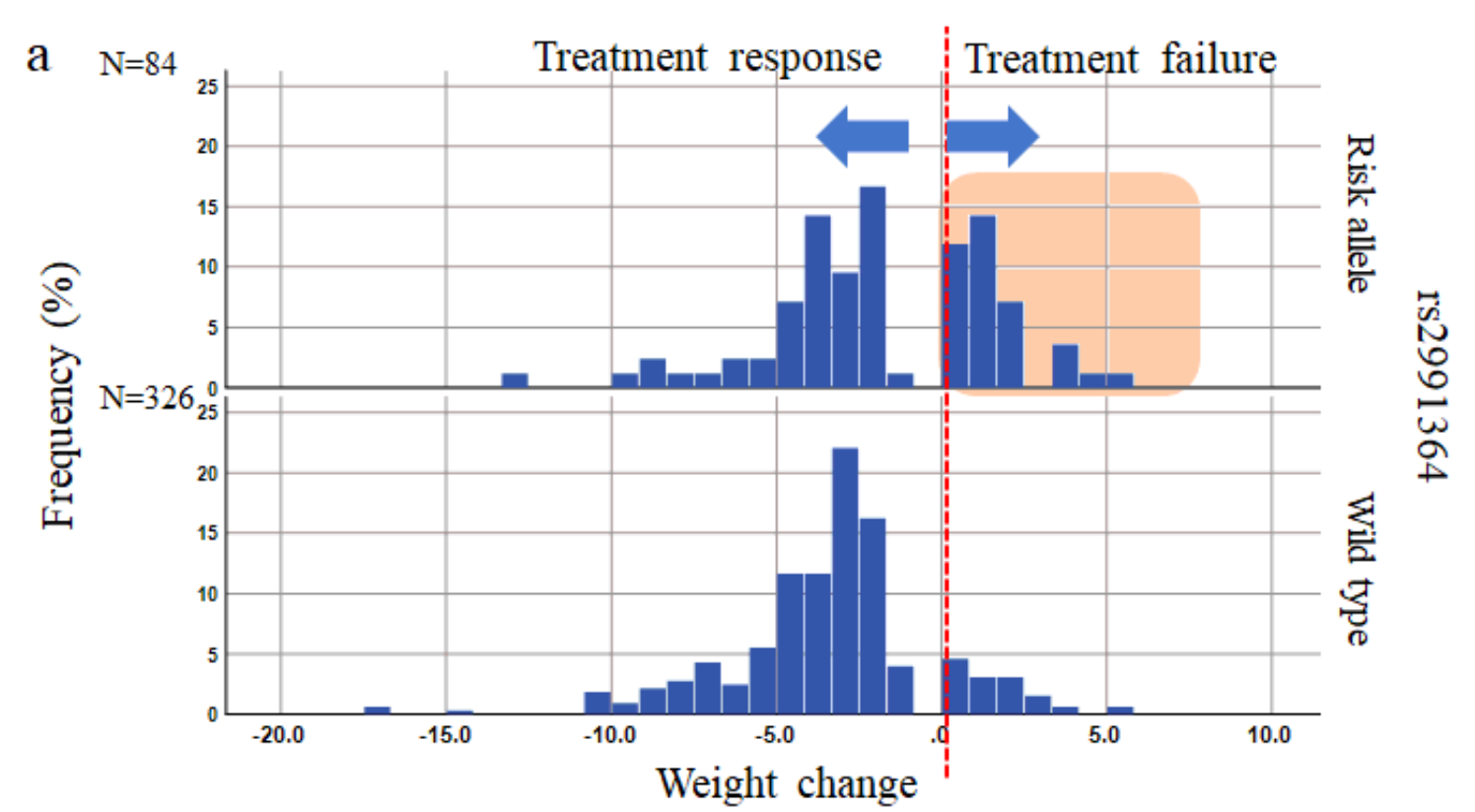

$\mathrm{b}$

\begin{tabular}{c|c|c|c} 
Parameter & P value & Beta & OR \\
BUN & 0.003 & 0.077 & $1.08(1.026-1.136)$ \\
\hline $\mathrm{Na}$ & 0.005 & -0.179 & $0.836(0.738-0.948)$ \\
\hline Constant & 0.009 & 22.234 & - \\
\hline
\end{tabular}

Score $=\mathbf{2 2 . 2 3 4}+\mathrm{BUN}^{*} 0.077+\mathrm{Na}^{*}(-0.179)$

Probability for treatment failure

$=\exp ($ score $) /\{1+\exp ($ score $)\}$

Optimal cutoff = probability of $38.6 \%$

Sensitivity $84.4 \%$

Specificity $\mathbf{7 0 . 0 \%}$ c

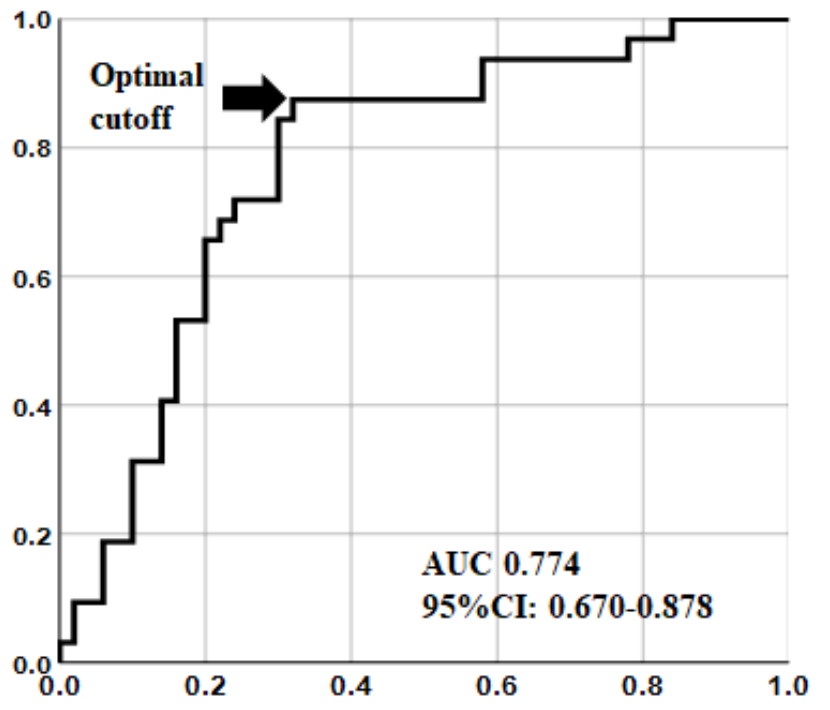

Figure 1

a) Histogram for weight change stratified with or without the rs2991364 risk allele. Treatment failure cases were concentrated in populations with risk alleles (red area). Prediction model construction and evaluation for treatment failure in a population with the rs2991364 risk allele. (b) Details of the prediction model based on logistic regression in which a backward elimination $(P<0.05)$ method was applied after including age, sex, weight, Child-Pugh classification, platelet, \%PT, albumin, AST, ALT, total bilirubin, BUN, creatinine, $\mathrm{Na}$, and etiology (HBV, HCV, alcohol, and NASH). (c) ROC analysis of the prediction model. 


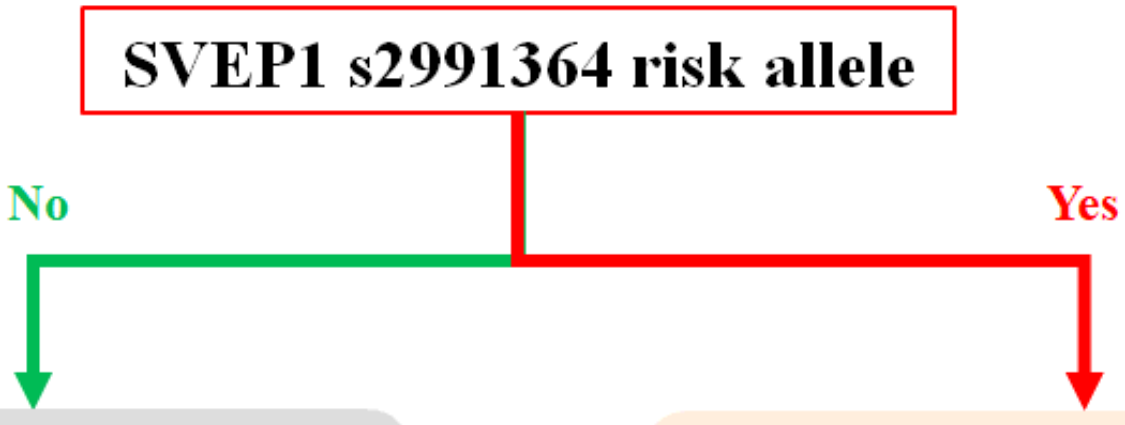

Treatment response

282/328

(86.0\%)
The probability of treatment failure : prediction model $>38.6 \%$

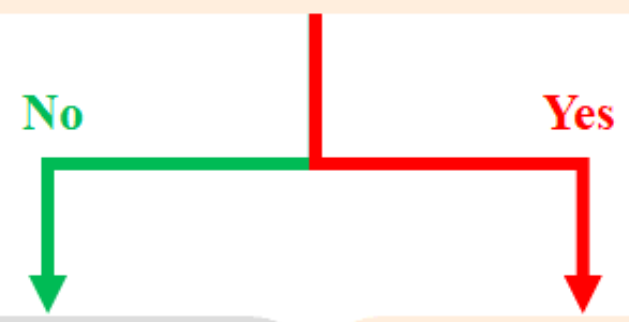

Treatment response

$35 / 40$

(87.5\%)
Treatment failure $27 / 42$ (64.3\%)

Figure 2

Diagnostic flow diagram for treatment failure based on the rs2991364 risk allele and the prediction model. This flow diagram can used to identify high-risk patients for tolvaptan treatment failure. 
Fig. 3

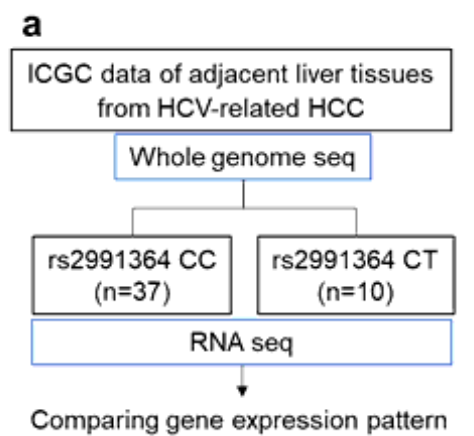

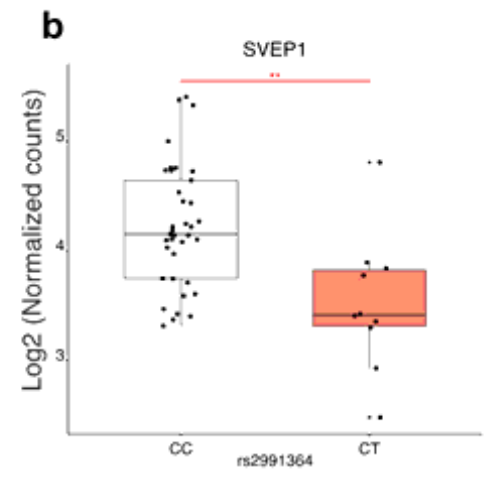

c

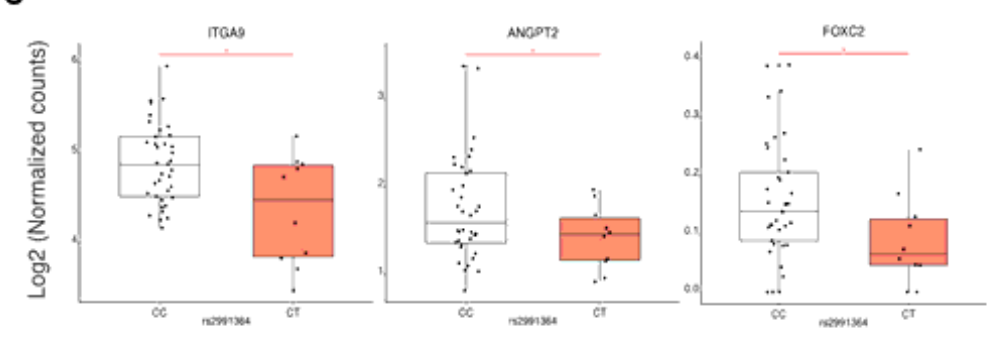

d

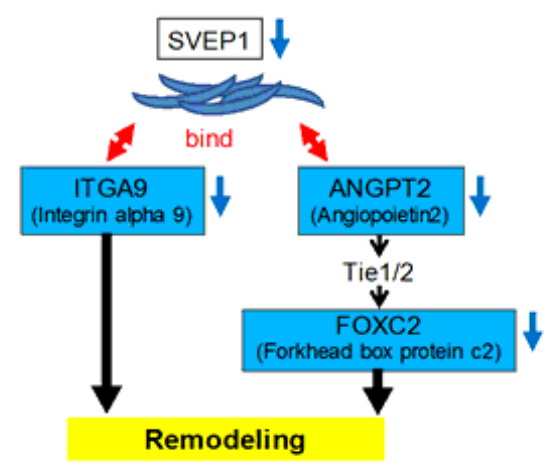

\section{Figure 3}

rs2991364 genotype affects the expression of SVEP1 and its downstream genes. (a) Workflow showing sampling, sequencing, and analysis process for ICGC liver data. (b) Impact of SNP rs2991364 genotypes on expression of the SVEP1 gene in ICGC data (TT-allele; $n=37, C T$-allele; $n=10)$. SVEP1 expression was downregulated in the minor CT-allele group $(P=6.70 \times 10-4)$. Data derived from RNA-seq. Mean and SEM are shown. Level of significance at $* P<0.05, * \star P<0.01$. (c) The effect of SNP rs2991364 genotypes on expression of SVEP1 downstream signal genes. ITGA9, ANGPT2, and FOXC2 were downregulated in the minor CT-allele group (ITGA9: $P=0.011$, ANGPT2: $P=0.025$, FOXC2: $P=0.015$ ). (d) Impact of SNP rs2991364 genotypes on SVEP1 signaling pathway. rs2991364 affects the expression of SVEP1 signal genes, resulting in vascular network fragility.

\section{Supplementary Files}

This is a list of supplementary files associated with this preprint. Click to download. 
- GraphicAbstract.tif

- FigS1.tif

- Figs2.tif

- FigS3.tif

- Fig.S4.tif

- Supplementarymanuscript202141.docx 